\title{
Whole-body vibration with extremely low-amplitude accelerates early-stage bone defect healing with reducing angiogenesis
}

\author{
Takeshi Matsumoto, Daichi Goto, Shota Sato \\ Bioengineering Division, Osaka University Graduate School of Engineering Science
}

Introduction

- Whole-body vibration (WBV) during bone healing

- Callus volume

- Mineral deposition

- Material property

(Leung et al: J Orthop Res 2009, Shi et al: Bone 2010, etc)

- Angiogenesis --- a requisite for fracture healing
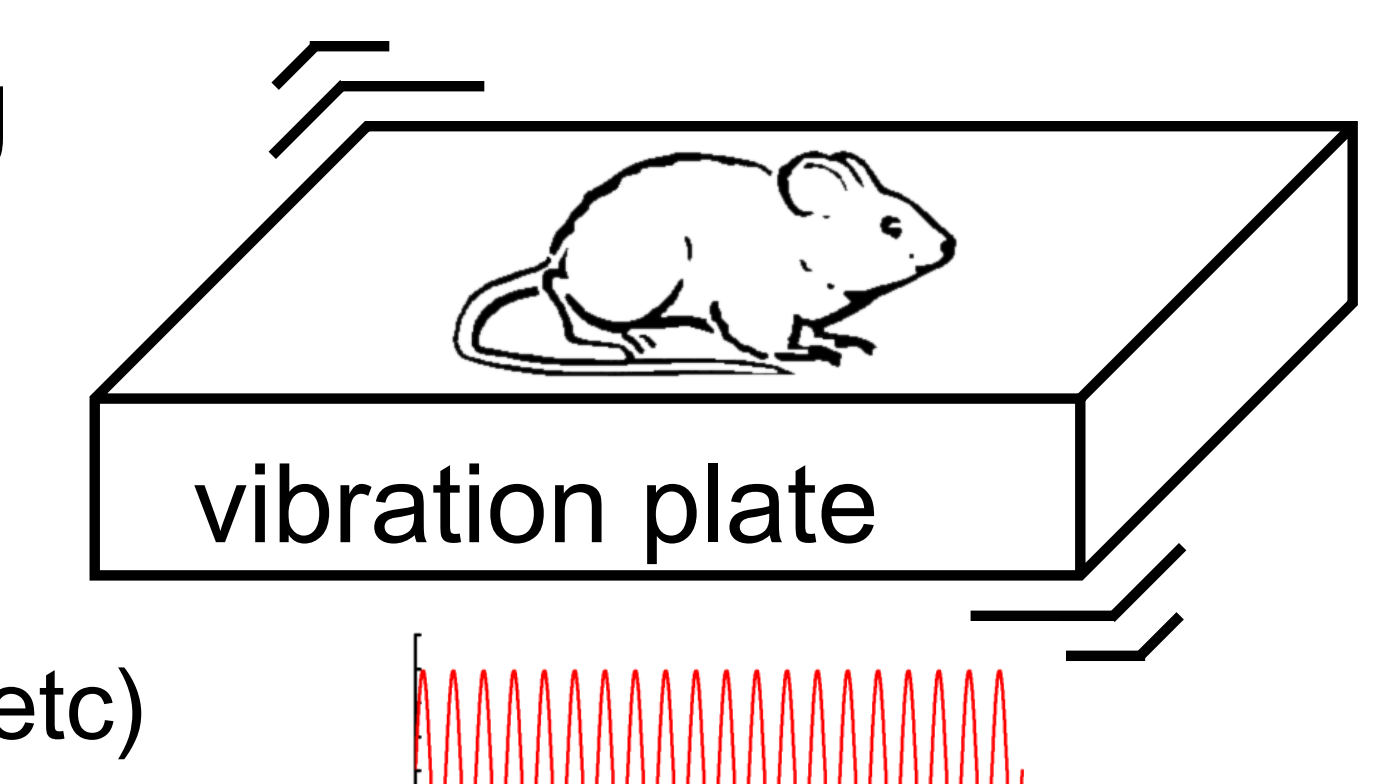

sinusoidal wave

\section{Aim}

To evaluate the effect of whole-body vibration (WBV) with extremely low-amplitude on early-stage bone defect healing, mainly focusing on the neo-vascular growth.

-WBV-load device

\section{Methods}
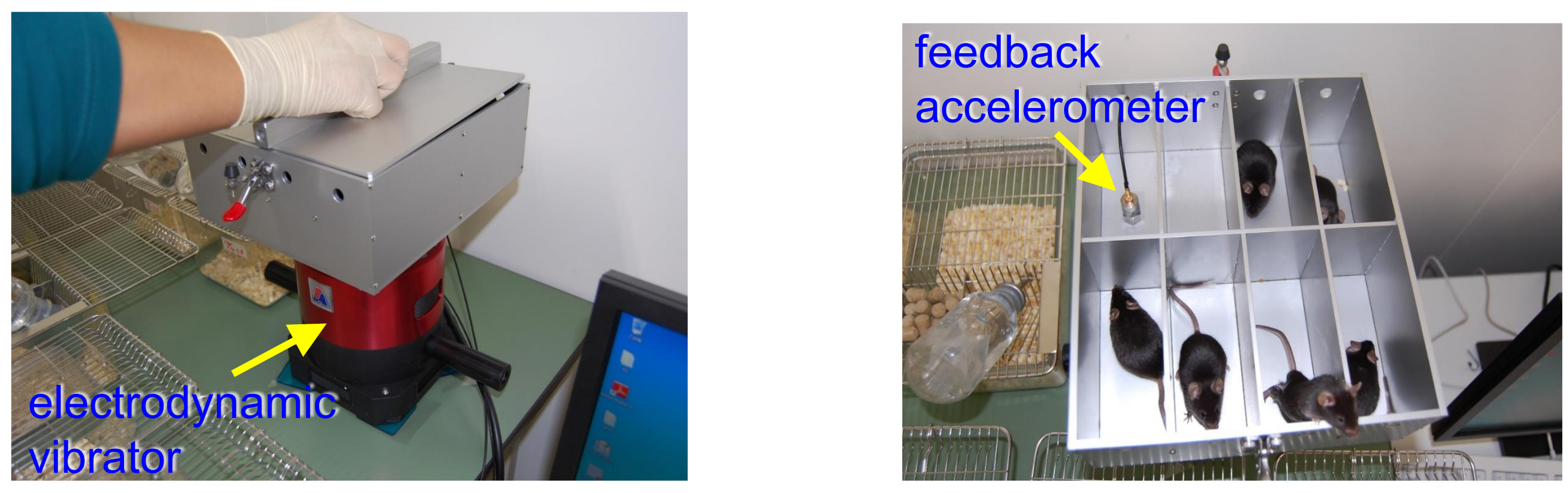

-Animals

C57BL/6J (ơ), 13 week old

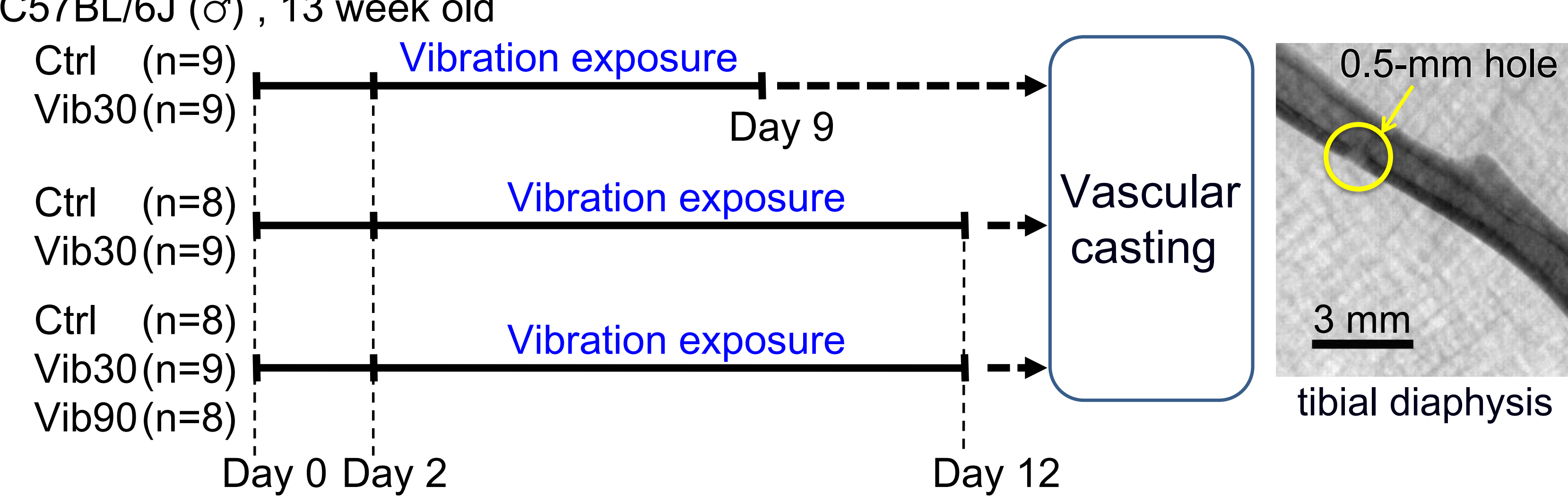

Vib30: $30-\mathrm{Hz}, 0.03 \mathrm{~g}$ (peak-to-peak) sinusoidal WBV, given daily for $20 \mathrm{~min} / \mathrm{d}$ Vib90: $90-\mathrm{Hz}, 0.03 \mathrm{~g}$ (peak-to-peak) sinusoidal WBV, given daily for $20 \mathrm{~min} / \mathrm{d}$ Ctrl: no vibration (only put in the WBV-load device )

\section{- Vascular casting}

Zirconia casting agent: mixture (1:2) of $9 \% \mathrm{v} / \mathrm{v}-\mathrm{ZrO}_{2}$ colloid and $1.2 \% \mathrm{w} / \mathrm{v}$-agarose Infusion Solidification
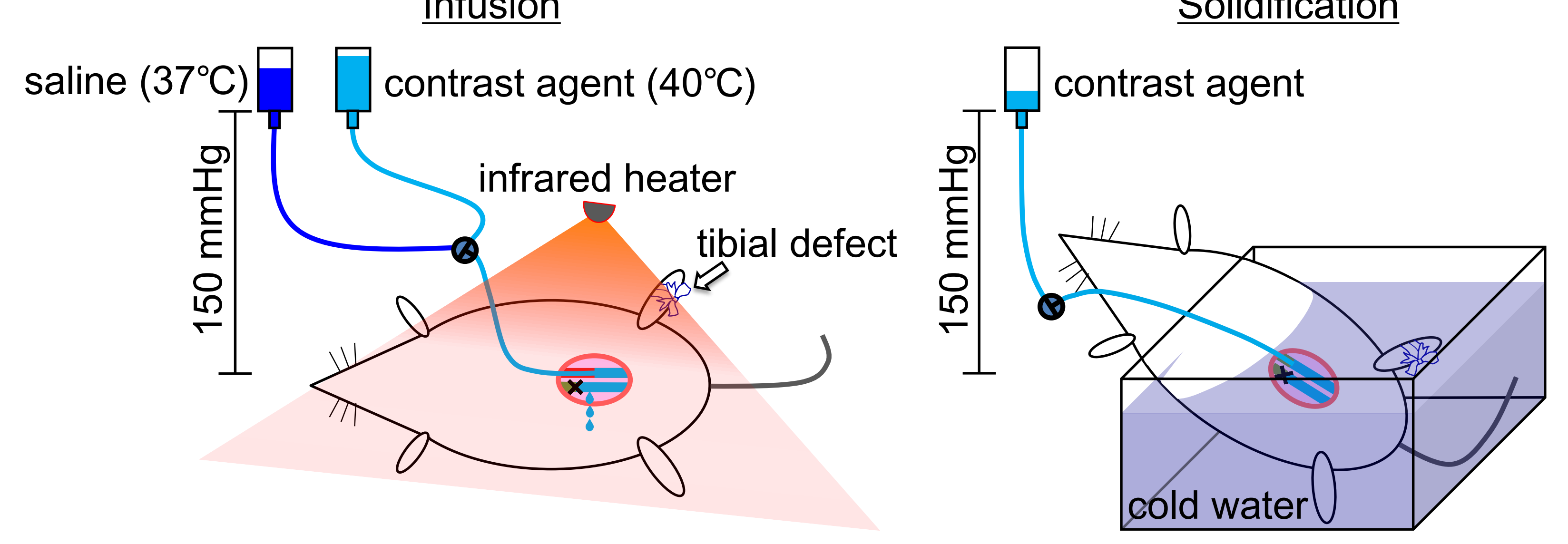

- Synchrotron CT
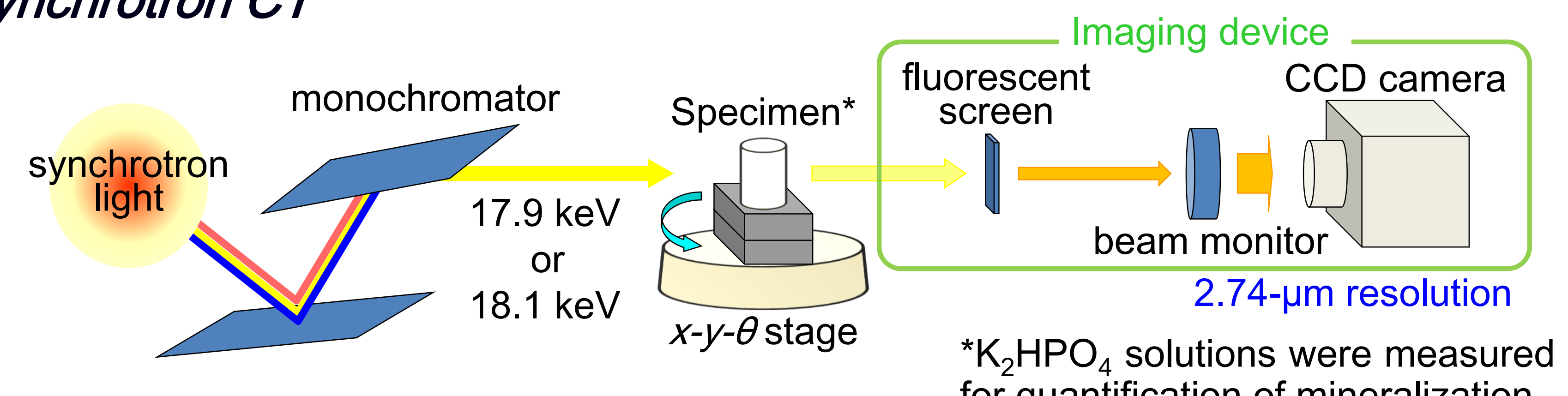

-Image analysis

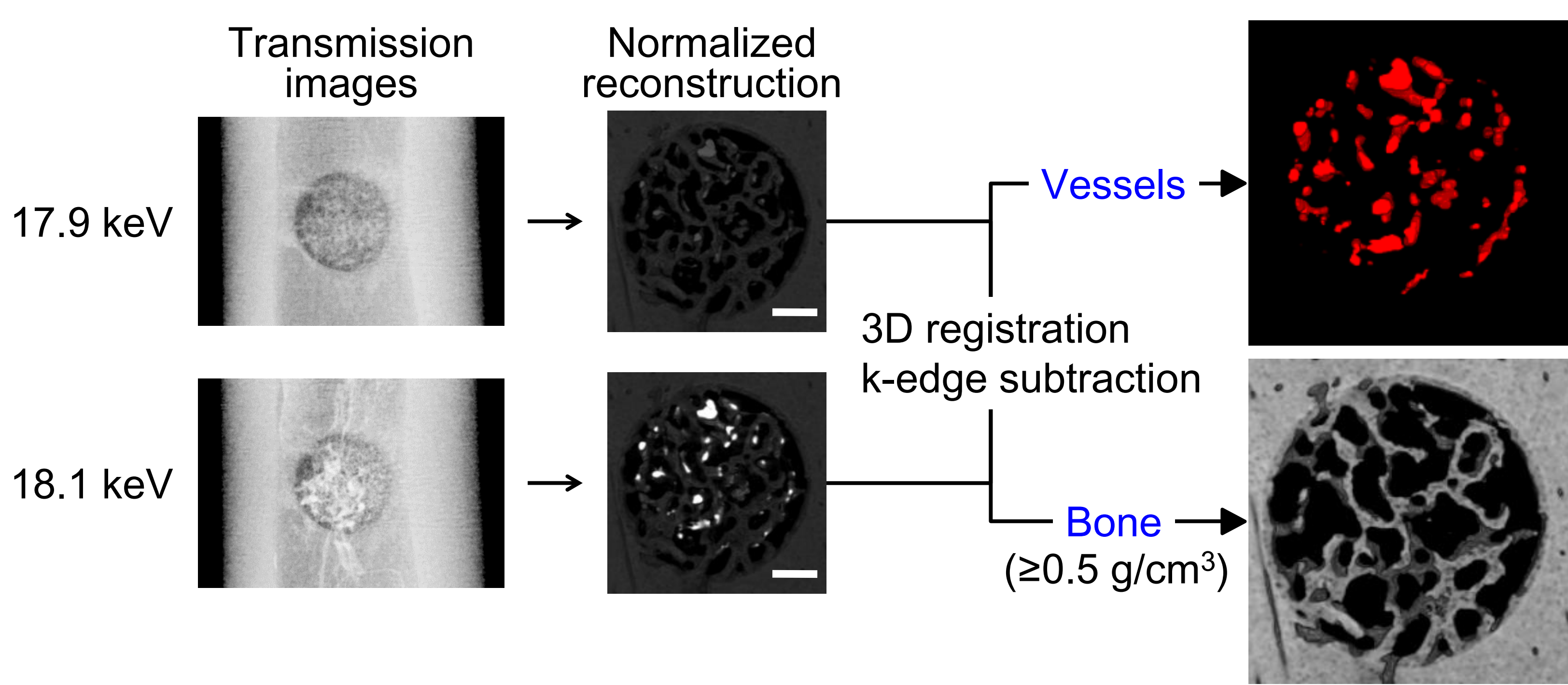

Results

Differences between age-matched groups were assessed by Kruskal-Wallis test followed by Dunn's multiple comparison test.

-3D displays of neovasculature and regenerated bone

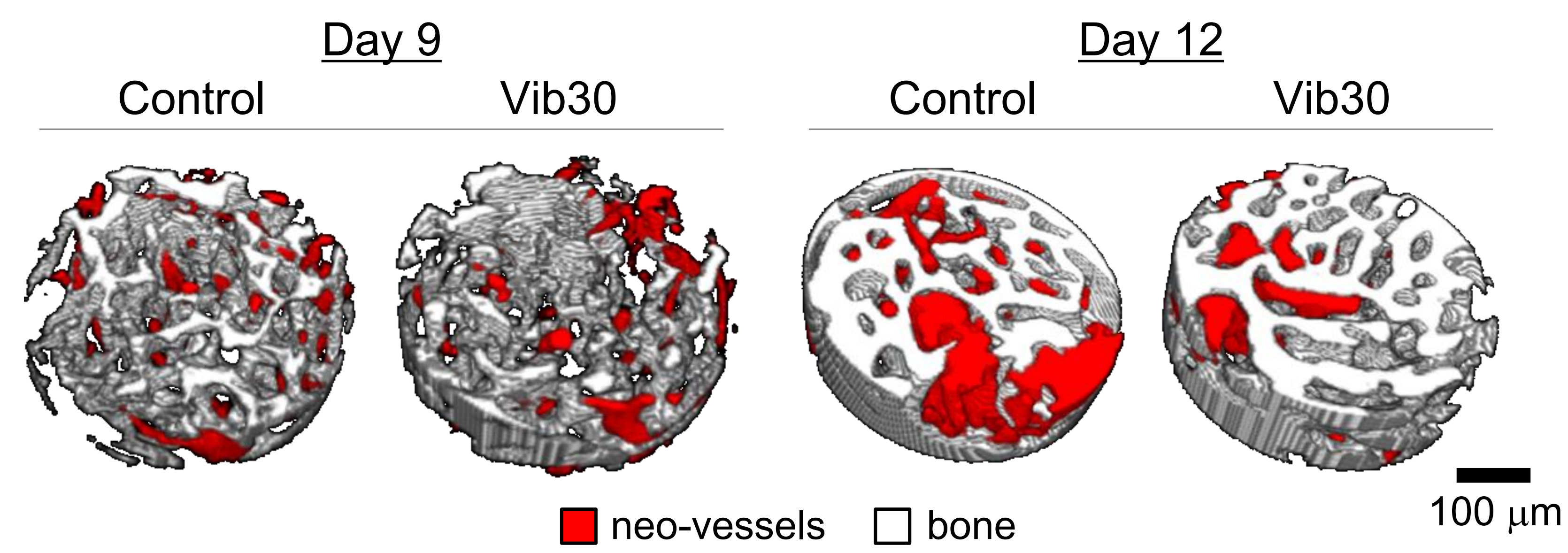

- Volume fraction
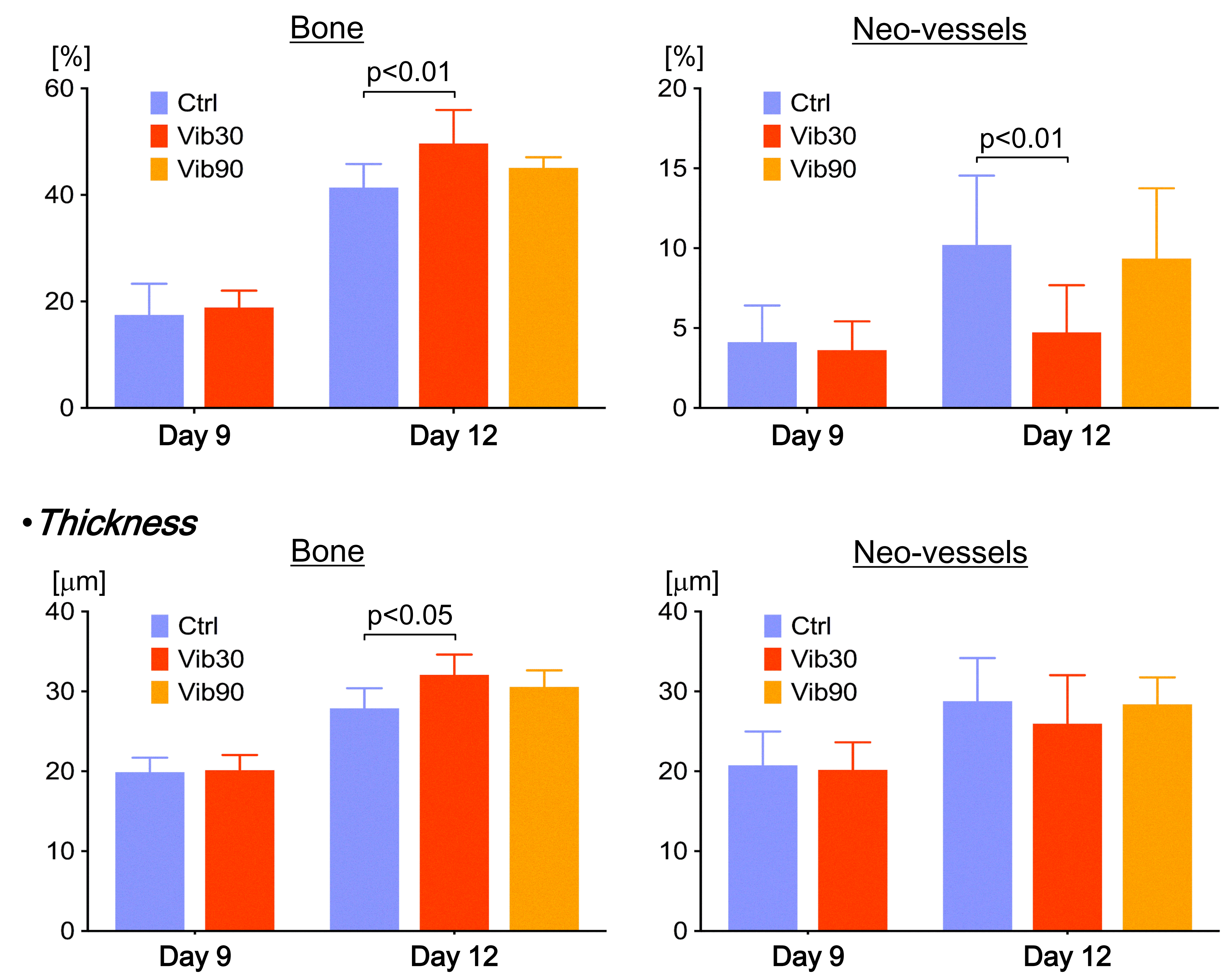

-Vessel segment frequency and length vs. diameter
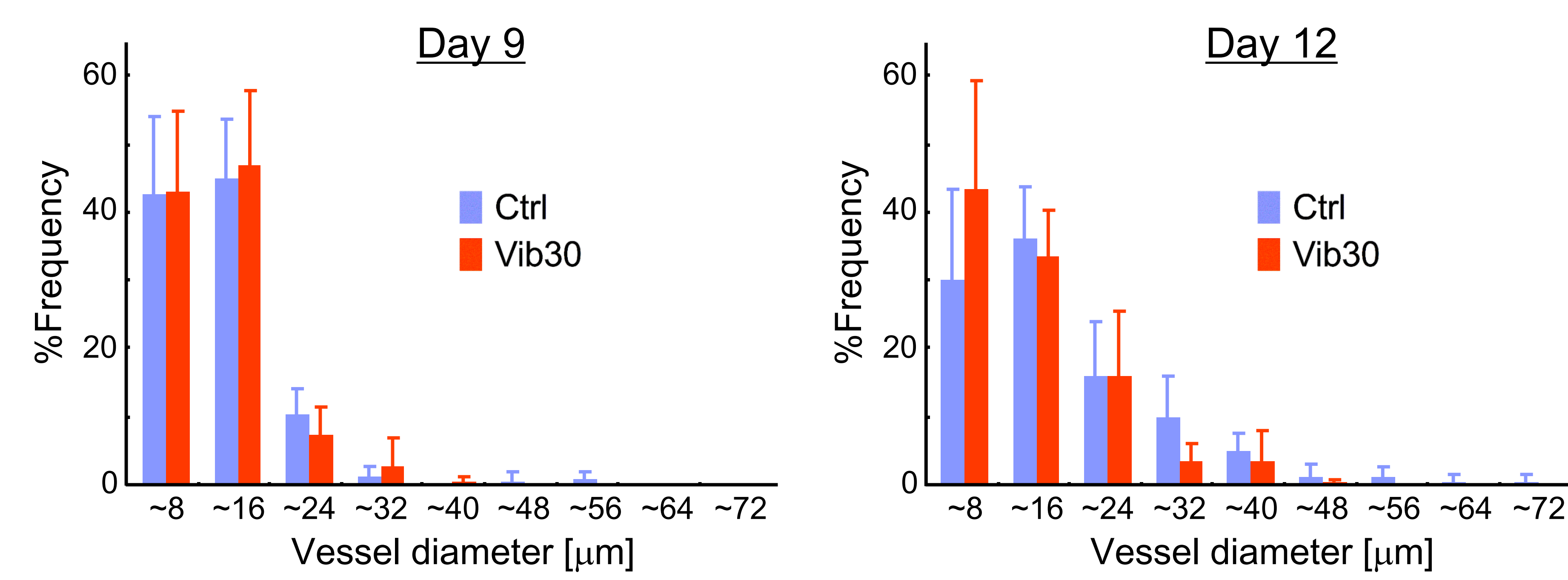

- Distribution of bone volume vs. density of hydroxyapatite on Day 12

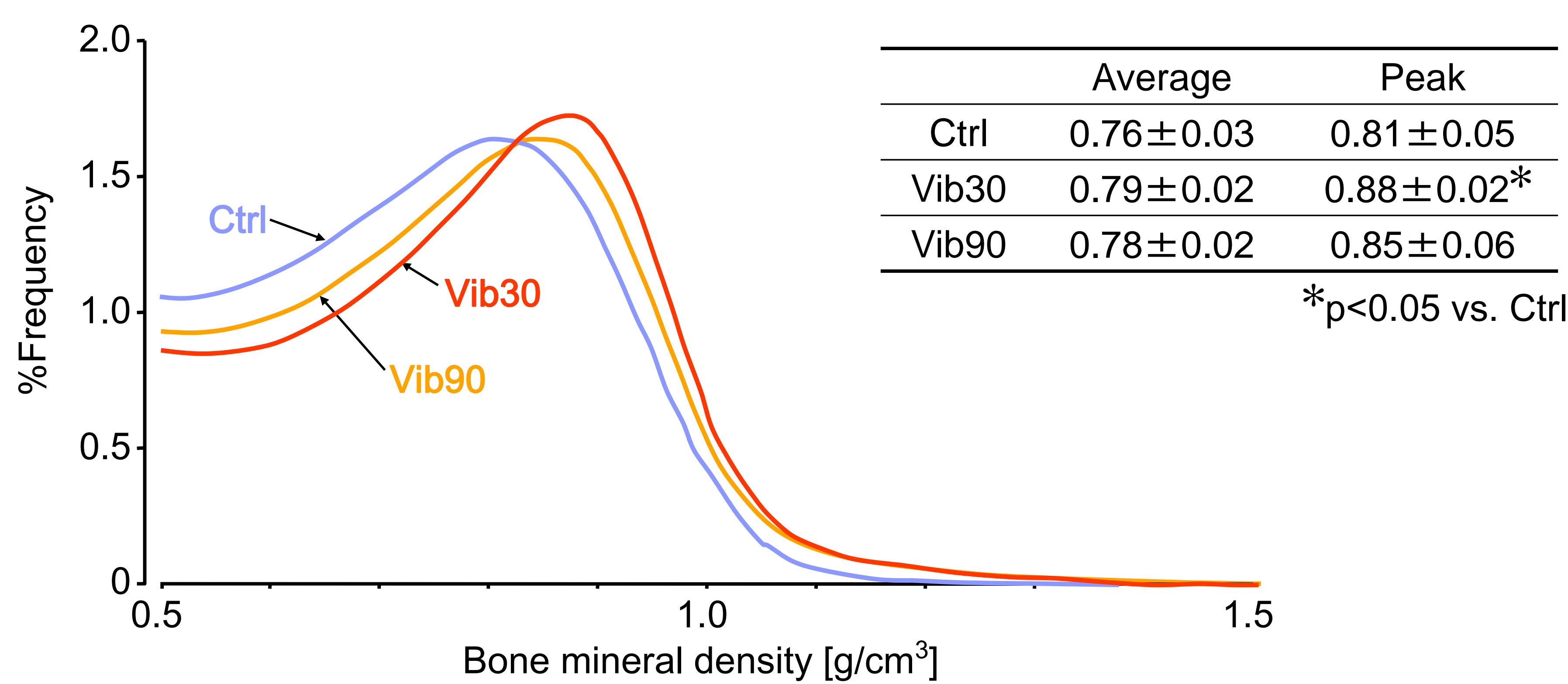

\section{Conclusions}

Extremely low-amplitude WBV facilitates early-stage bone defect repair. Reduced angiogenesis, which would still be suffice to promote osteogenesis, implies that the present WBV leads to preferred differentiation of mesenchymal stem cells into osteoblastic cells instead of differentiating toward an endothelial lineage. 